\title{
An inverse problem of reconstructing the time-dependent coefficient in a one-dimensional hyperbolic equation
}

\author{
M.J. Huntul ${ }^{1 *}$, Muhammad Abbas ${ }^{2 *}$ (D) and Dumitru Baleanu $u^{3,4,5}$
}

\author{
"Correspondence: \\ mhantool@jazanu.edu.sa; \\ muhammad.abbas@uos.edu.pk \\ 'Department of Mathematics, \\ Faculty of Science, Jazan University, \\ Jazan, Saudi Arabia \\ 2Department of Mathematics, \\ University of Sargodha, 40100 \\ Sargodha, Pakistan \\ Full list of author information is \\ available at the end of the article
}

\begin{abstract}
In this paper, for the first time the inverse problem of reconstructing the time-dependent potential (TDP) and displacement distribution in the hyperbolic problem with periodic boundary conditions (BCs) and nonlocal initial supplemented by over-determination measurement is numerically investigated. Though the inverse problem under consideration is ill-posed by being unstable to noise in the input data, it has a unique solution. The Crank-Nicolson-finite difference method (CN-FDM) along with the Tikhonov regularization (TR) is applied for calculating an accurate and stable numerical solution. The programming language MATLAB built-in Isqnonlin is used to solve the obtained nonlinear minimization problem. The simulated noisy input data can be inverted by both analytical and numerically simulated. The obtained results show that they are accurate and stable. The stability analysis is performed by using Fourier series.
\end{abstract}

Keywords: Hyperbolic equation; Inverse problem; Periodic boundary; Integral boundary; Tikhonov regularization; Optimization

\section{Introduction}

The reconstruction of the unknown coefficients in the inverse problem of the hyperbolic problem has various applications in science and engineering. In the last few decades, various authors have reconstructed the unknown coefficients in the inverse problem of the hyperbolic wave equations. For example, Bakushinsky and Leonov [2] recovered the spacedependent coefficient from integral data, while authors of $[5,24,25]$ determined the timedependent source coefficients. Cannon and Dunninger [7] reconstructed a force function from over-specified data. Further, Cannon and DuChateau [6] determined both timeand space-dependent coefficients. Stefanov and Uhlmann [27] recovered a source term in anisotropic media. Additionally, Bellassoued and Yamamoto [3] studied the inverse problem to determine the unknown term in the hyperbolic model with variable terms. Boumenir and Tuan [4] showed the process for reconstructing the unknown coefficient in the inverse problem of the wave equation from a finite number of special lateral measurements. Yamamoto [31] considered an inverse problem for identifying space-dependent function from Neumann BCs and showed a TR and reconstruction formula. Yang et al.

(c) The Author(s) 2021. This article is licensed under a Creative Commons Attribution 4.0 International License, which permits use, sharing, adaptation, distribution and reproduction in any medium or format, as long as you give appropriate credit to the original author(s) and the source, provide a link to the Creative Commons licence, and indicate if changes were made. The images or other third party material in this article are included in the article's Creative Commons licence, unless indicated otherwise in a credit line to the material. If material is not included in the article's Creative Commons licence and your intended use is not permitted by statutory regulation or exceeds the permitted use, you will need to obtain permission directly from the copyright holder. To view a copy of this licence, visit http://creativecommons.org/licenses/by/4.0/. 
[32] recovered heat source term in the inverse problem of heat conduction equation using Tikhonov regularization. Huntul [12] identified the unknown time-dependent coefficient in the third-order equation from nonlocal integral observation.

Recently, the inverse problems of the wave equations for recovering time-dependent potential from over-determination integral condition have been investigated by Tekin [29] while the time-dependent force function has been studied by Hussein and Lesnic [19]. The authors of $[13-15,17]$ studied the inverse problems for identifying the time-wise potential terms in third and fourth-order equations. Huntul et al. [18] investigated an inverse problem to reconstruct the time-wise potential terms in a wave equation as an overdetermination condition. In $[10,11,20]$, authors recovered the time- and space-dependent source functions. Huntul and Tamsir [16] investigated an inverse problem to recover a time-wise heat source from the integral condition. Still, the inverse problem of reconstructing the time-wise potential coefficient numerically for the hyperbolic problems with integral and periodic BCs is inadequate in the literature.

In this paper, the time-dependent potential is reconstructed for the first time numerically in a one-dimensional hyperbolic problem with periodic and integral $\mathrm{BCs}$ from the over-determination estimation. Azizbayov [1] has already proved that this problem is locally uniquely solvable, but no numerical realization has been carried out till now, which is the main contribution of proposed work.

The paper is organized as follows: The research problem is stated in Sect. 2. Sect. 3 discretizes the direct problem using CN-FDM. Stability analysis is presented in Sect. 4, while the minimization technique is given in Sect. 5. Some numerical results are tabulated in Sect. 6, while the conclusions are highlighted in Sect. 7.

\section{Formulation of the inverse problem}

We consider an inverse problem of reconstructing an unknown time-dependent potential coefficient $\alpha(t)$ in the one-dimensional hyperbolic equation

$$
\frac{\partial^{2} z}{\partial t^{2}}-\frac{\partial^{2} z}{\partial r^{2}}=\alpha(t) z+s(r, t), \quad(r, t) \in D_{T}:=(0,1) \times(0, T),
$$

where $z=z(r, t)$ is an unknown displacement, $s(r, t)$ is a known source term which is as distributed force. The nonlocal initial conditions (ICds)

$$
z(r, 0)=\int_{0}^{T} P_{1}(t) z(r, t) d t+\xi(r), \quad z_{t}(r, 0)=\int_{0}^{T} P_{2}(t) z(r, t) d t+\zeta(r), \quad r \in[0,1],
$$

the periodic $\mathrm{BCs}$

$$
z(0, t)=z(1, t), \quad t \in[0, T]
$$

the integral boundary condition

$$
\int_{0}^{1} z(r, t) d r=0, \quad t \in[0, T]
$$

and the over-determination condition

$$
z\left(R_{0}, t\right)=q(t), \quad t \in[0, T],
$$


where $R_{0} \in(0,1)$ is some fixed point, the functions $P_{1}(t), P_{2}(t), \xi(r), \zeta(r)$, and $q(t)$ are given.

The numerical solution of the inverse problem hyperbolic wave equation (1)-(5) is written as $\{\alpha(t), z(r, t)\}$ such that $\alpha(t) \in C[0, T]$ and $z(r, t) \in C^{2}\left(\bar{D}_{T}\right)$. Along with inverse problem (1)-(5), the following auxiliary inverse problem is considered. It is needed to find $\{\alpha(t), z(r, t)\} \in C[0, T] \times C^{2}\left(\bar{D}_{T}\right)$ from (1)-(3), and

$$
\begin{aligned}
& z_{r}(0, t)=z_{r}(1, t), \quad t \in[0, T], \\
& q^{\prime \prime}(t)-z_{r r}\left(R_{0}, t\right)=\alpha(t) q(t)+s\left(R_{0}, t\right), \quad t \in[0, T] .
\end{aligned}
$$

The upcoming theorems are taken from [1], and they read as follows.

Theorem 1 Suppose that $\xi(r), \zeta(r) \in C[0,1], P_{i}(t) \in C[0, T], i=1,2, q(t) \in C^{2}[0, T], q(t) \neq$ $0, s(r, t) \in C\left(\bar{D}_{T}\right), \int_{0}^{1} s(r, t) d r=0, t \in[0, T]$, and the consistency conditions, given below, are satisfied:

$$
\begin{aligned}
& \int_{0}^{1} \xi(r) d r=0, \quad \int_{0}^{1} \zeta(r) d r=0, \\
& q(0)=\int_{0}^{T} q(t) P_{1}(t) d t+\xi\left(R_{0}\right), \quad q^{\prime}(0)=\int_{0}^{T} q(t) P_{2}(t) d t+\zeta\left(R_{0}\right) .
\end{aligned}
$$

\section{Then the following arguments are true:}

1. Each classical solution $(\alpha(t), z(r, t))$ of (1)-(5) is a solution of (1)-(3), (6), and (7);

2. Each solution $(\alpha(t), z(r, t))$ of (1)-(3), (6), and (7) by virtue of

$$
\left(T\left\|P_{2}(t)\right\|_{C[0, T]}+T\left\|P_{1}(t)\right\|_{C[0, T]}+\frac{T}{2}\|\alpha(t)\|_{C[0, T]}\right) T<1
$$

is a classical solution of (1)-(5).

We impose the below conditions to the functions $\xi, \zeta, s, P_{1}, P_{2}$, and $q$ [1]:

(A1) $\xi^{\prime \prime \prime}(r) \in L_{2}(0,1), \xi(r) \in C^{2}[0,1], \xi(0)=\xi(1), \xi^{\prime}(0)=\xi^{\prime}(1), \xi^{\prime \prime}(0)=\xi^{\prime \prime}(1)$;

(A2) $\zeta^{\prime \prime}(r) \in L_{2}(0,1), \zeta(r) \in C^{1}[0,1], \zeta(0)=\zeta(1), \zeta^{\prime}(0)=\zeta^{\prime}(1)$;

(A3) $s(r, t), s_{r}(r, t) \in C\left(\bar{D}_{T}\right), s_{r r}(r, t) \in L_{2}\left(\bar{D}_{T}\right), s(0, t)=s(1, t), s_{r}(0, t)=s_{r}(1, t), t \in[0, T]$;

(A4) $P_{1}(t), P_{2}(t) \in C[0, T], q(t) \in C^{2}[0, T], q(t) \neq 0, t \in[0, T]$.

Theorem 2 Let conditions (A1)-(A4) be satisfied, and suppose that

$$
\left(\left(A_{1}(T)+A_{2}(T)+2\right)\left(B_{1}(T)+B_{2}(T)\right)+C_{1}(T)+C_{2}(T)\right)\left(A_{1}(T)+A_{2}(T)+2\right)<1,
$$

where

$$
\begin{aligned}
A_{1}(T)= & T\|\zeta(r)\|_{L_{2}(0,1)}+T \sqrt{T}\|s(r, t)\|_{L_{2}\left(\bar{D}_{T}\right)}+\|\xi(r)\|_{L_{2}(0,1)}+2 \sqrt{6}\left\|\xi^{\prime \prime \prime}(r)\right\|_{L_{2}(0,1)} \\
& +2 \sqrt{6 T}\left\|s_{r}(r, t)\right\|_{L_{2}\left(\bar{D}_{T}\right)}+2 \sqrt{6}\left\|\zeta^{\prime \prime}(r)\right\|_{L_{2}(0,1)},
\end{aligned}
$$




$$
\begin{aligned}
A_{2}(T)= & \left\|q^{-1}(t)\right\|_{C[0, T]}\left\{\left\|q^{\prime \prime}(t)-s\left(R_{0}, t\right)\right\|_{C[0, T]}+2\left(\sum_{k=1}^{\infty} \lambda_{k}^{-2}\right)^{\frac{1}{2}} \times\left[\left\|\xi^{\prime \prime \prime}(r)\right\|_{L_{2}(0,1)}\right.\right. \\
& \left.\left.+\left\|\zeta^{\prime \prime}(r)\right\|_{L_{2}(0,1)}+\sqrt{T}\left\|s_{r}(r, t)\right\|_{L_{2}\left(\bar{D}_{T}\right)}\right]\right\}, \quad B_{1}(T)=T^{2}+2 \sqrt{6} T, \\
B_{2}(T)= & \left\|q^{-1}(t)\right\|_{C[0, T]}\left(\sum_{k=1}^{\infty} \lambda_{k}^{-2}\right)^{\frac{1}{2}} T, \\
C_{1}(T)= & T(1+2 \sqrt{6})\left\|P_{1}(t)\right\|_{C[0, T]}+T(T+2 \sqrt{6})\left\|P_{2}(t)\right\|_{C[0, T]}, \\
C_{2}(T)= & 2\left\|q^{-1}(t)\right\|_{C[0, T]}\left(\sum_{k=1}^{\infty} \lambda_{k}^{-2}\right)^{\frac{1}{2}} T\left(\left\|P_{2}(t)\right\|_{C[0, T]}+T\left\|P_{1}\right\|_{C[0, T]}\right) .
\end{aligned}
$$

Then inverse problem (1)-(3), (6), (7) has a unique solution in the ball $K=K_{R}$.

The uniqueness of solution for inverse problem (1)-(5) has been proved by Azizbayov [1]; it is given as follows.

\section{Theorem 3 Let the conditions}

$$
\begin{aligned}
& \int_{0}^{1} \xi(r) d r=0, \quad \int_{0}^{1} \zeta(r) d r=0, \quad \int_{0}^{1} s(r, t) d r=0, \quad t \in[0, T], \\
& q(0)=\int_{0}^{T} P_{1}(t) q(t) d t+\xi\left(R_{0}\right), \quad q^{\prime}(0)=\int_{0}^{T} P_{2}(t) q(t) d t+\zeta\left(R_{0}\right), \\
& \left(T\left\|P_{2}(t)\right\|_{C[0, T]}+T\left\|P_{1}(t)\right\|_{C[0, T]}+\frac{T}{2}\left(A_{1}(T)+A_{2}(T)+2\right)\right) T<1,
\end{aligned}
$$

and all the assumptions of Theorem 2 be satisfied. Then (1)-(5) has a unique solution in $K=K_{R}\left(\|z\|_{E_{T}^{3}} \leq A_{1}(t)+A_{2}(t)+2\right)$ of the space $E_{T}^{3}$.

\section{Numerical solution of the forward problem}

First of all, we consider forward problem (1)-(4) with all initial and boundary conditions, then we calculate solution using the CN-FDM scheme in this section.

Theorem 4 Let $\alpha(t), \xi(r), \zeta(r), P_{1}(t), P_{2}(t), s(r, t)$ be known functions, and the CN-FDM scheme is utilized for time discretization. Then the numerical solution $z(r, t)$ is given in equations (19) and (21).

Proof We denote $z\left(r_{i}, t_{j}\right)=z_{i, j}, P_{1}(t)=P_{1}^{j}, P_{2}(t)=P_{2}^{j}, s\left(r_{i}, t_{j}\right)=s_{i, j}$, and $\alpha\left(t_{j}\right)=\alpha_{j}$, where $r_{i}=$ $i \Delta r, t_{j}=j \Delta t, \Delta r=\frac{1}{M}$, and $\Delta t=\frac{T}{N}, i=0(1) M$ and $j=0(1) N$. Then the $\left(\Delta r^{2}, \Delta t^{2}\right)$ CN-FDM [26] discretizes (1) as follows:

$$
\begin{gathered}
\frac{z_{i, j+1}-2 z_{i, j}+z_{i, j-1}}{(\Delta t)^{2}}-\left(\frac{z_{i+1, j+1}-2 z_{i, j+1}+z_{i-1, j+1}+z_{i+1, j}-2 z_{i, j}+z_{i-1, j}}{2(\Delta r)^{2}}\right) \\
=\frac{\alpha_{j+1}}{2} z_{i, j+1}+\frac{\alpha_{j}}{2} z_{i, j}+\frac{1}{2}\left(s_{i, j+1}+s_{i, j}\right), \quad i=1(1) M, j=1(1) N .
\end{gathered}
$$


Equation (11) yields

$$
\begin{aligned}
& -A z_{i-1, j+1}+\left(1+2 A-B_{j+1}\right) z_{i, j+1}-A z_{i+1, j+1} \\
& =A z_{i-1, j}+\left(2+2 A+B_{j}\right) z_{i, j}+A z_{i+1, j}-z_{i, j-1} \\
& \quad+\frac{(\Delta t)^{2}}{2}\left(s_{i, j+1}+s_{i, j}\right), \quad i=1(1) M, j=1(1) N,
\end{aligned}
$$

where

$$
A=\frac{(\Delta t)^{2}}{2(\Delta r)^{2}}, \quad B_{j}=\frac{(\Delta t)^{2}}{2} \alpha_{j}
$$

The discretization of nonlocal ICs (2) is

$$
\begin{aligned}
& z_{i, 0}=\frac{\Delta t}{3}\left(P_{1}^{0} z_{i, 0}+P_{1}^{N} z_{i, N}+\sum_{l=1,3}^{N-1} P_{1}^{l} z_{i, l}+\sum_{l=2,4}^{N-2} P_{1}^{l} z_{i, l}\right)+\xi\left(r_{i}\right), \quad i=0(1) M, \\
& \frac{z_{i, 1}-z_{i,-1}}{2(\Delta t)}=\frac{\Delta t}{3}\left(P_{2}^{0} z_{i, 0}+P_{2}^{N} z_{i, N}+\sum_{l=1,3}^{N-1} P_{2}^{l} z_{i, l}+\sum_{l=2,4}^{N-2} P_{2}^{l} z_{i, l}\right)+\zeta\left(r_{i}\right), \quad i=0(1) M,
\end{aligned}
$$

and the periodic $\mathrm{BC}(3)$ is

$$
z(0, t)=z_{0, j}=z(1, t)=z_{M, j} .
$$

Finally, discretization of integral BC (4) is given as follows:

$$
\int_{0}^{1} z\left(r, t_{j}\right) d r=\frac{\Delta r}{3}\left(z_{0, j}+z_{M, j}+\sum_{l=1,3}^{M-1} z_{l, j}+\sum_{l=2,4}^{M-2} z_{l, j}\right)=0, \quad j=0(1) N .
$$

For $i=0$ and $M$, from equations (15) and (16), we get

$$
2 z_{0, j}+4 \sum_{l=1,3}^{M-1} z_{l, j}+2 \sum_{l=2,4}^{M-2} z_{l, j}=0, \quad i=0, j=0(1) N
$$

and

$$
4 \sum_{l=1,3}^{M-1} z_{l, j}+2 \sum_{l=2,4}^{M-2} z_{l, j}+2 z_{M, j}=0, \quad i=M, j=0(1) N
$$


The above equations (12), (17), and (18) can be reformulated and converted at time $t_{j+1}$ into the $(M+1) \times(M+1)$ system:

$$
\left[\begin{array}{cccccccc}
2 & 4 & 2 & 4 & 2 & \ldots & 4 & 0 \\
-A & \bar{B} & -A & 0 & 0 & \ldots & 0 & 0 \\
0 & -A & \bar{B} & -A & 0 & \ldots & 0 & 0 \\
\vdots & \ddots & \ddots & \ddots & \vdots & \ldots & \vdots & \vdots \\
0 & 0 & \cdots & -A & \bar{B} & -A & 0 & 0 \\
0 & 0 & \ldots & 0 & -A & \bar{B} & -A & 0 \\
0 & 0 & \ldots & 0 & 0 & -A & \bar{B} & -A \\
0 & 4 & \ldots & 4 & 2 & \ldots & 4 & 2
\end{array}\right]\left[\begin{array}{c}
z_{0, j+1} \\
z_{1, j+1} \\
z_{2, j+1} \\
z_{3, j+1} \\
\vdots \\
z_{M-2, j+1} \\
z_{M-1, j+1} \\
z_{M, j+1}
\end{array}\right]=\left[\begin{array}{c}
R_{0, j} \\
R_{1, j} \\
R_{2, j} \\
R_{3, j} \\
\vdots \\
R_{M-2, j} \\
R_{M-1, j} \\
R_{M, j}
\end{array}\right],
$$

where

$$
\begin{aligned}
\hat{B}=1 & +2 A-B_{j+1}, \quad R_{0, j}=R_{M, j}=0, \\
R_{i, j}= & A z_{i-1, j}+\left(2+2 A+B_{j}\right) z_{i, j}+A z_{i+1, j}-z_{i, j-1} \\
& +\frac{(\Delta t)^{2}}{2}\left(s_{i, j+1}+s_{i, j}\right), \quad i=1(1) M-1, j=1(1) N .
\end{aligned}
$$

At time $t_{1}$, using (14) in (12), we get

$$
\begin{aligned}
- & A z_{i-1,1}+\left(2+2 A-B_{1}\right) z_{i, 1}-A z_{i+1,1} \\
= & A z_{i-1,0}+\left(2+2 A+B_{0}\right) z_{i, 0}+A z_{i+1,0}+2(\Delta t) \zeta\left(r_{i}\right) \\
& +\frac{k^{2}}{3}\left(P_{2}^{0} z_{i, 0}+P_{2}^{N} z_{i, N}+\sum_{l=1,3}^{N-1} P_{2}^{l} z_{i, l}+\sum_{l=2,4}^{N-2} P_{2}^{l} z_{i, l}\right) \\
& +\frac{(\Delta t)^{2}}{2}\left(s_{i, 1}+s_{i, 0}\right), \quad i=1(1) M-1 .
\end{aligned}
$$

Now, at time $t_{1}$, equations (17), (18), and (20) can be reformulated into the $(M+1) \times(M+1)$ system:

$$
\left[\begin{array}{cccccccc}
2 & 4 & 2 & 4 & 2 & \ldots & 4 & 0 \\
-A & \hat{B} & -A & 0 & 0 & \ldots & 0 & 0 \\
0 & -A & \hat{B} & -A & 0 & \ldots & 0 & 0 \\
\vdots & \ddots & \ddots & \ddots & \vdots & \ldots & \vdots & \vdots \\
0 & 0 & \cdots & -A & \hat{B} & -A & 0 & 0 \\
0 & 0 & \cdots & 0 & -A & \hat{B} & -A & 0 \\
0 & 0 & \ldots & 0 & 0 & -A & \hat{B} & -A \\
0 & 4 & \ldots & 4 & 2 & \ldots & 4 & 2
\end{array}\right]\left[\begin{array}{c}
z_{0,1} \\
z_{1,1} \\
z_{2,1} \\
z_{3,1} \\
\vdots \\
z_{M-2,1} \\
z_{M-1,1} \\
z_{M, 1}
\end{array}\right]=\left[\begin{array}{c}
R_{0,0} \\
R_{1,0} \\
R_{2,0} \\
R_{3,0} \\
\vdots \\
R_{M-2,0} \\
R_{M-1,0} \\
R_{M, 0}
\end{array}\right]
$$

where

$$
\begin{aligned}
& \hat{B}=2+2 A-B_{1}, \quad R_{0,0}=R_{M, 0}=0, \\
& R_{i, 0}=A z_{i-1,0}+\left(2+2 A+B_{0}\right) z_{i, 0}+A z_{i+1,0}+2(\Delta t) \zeta\left(r_{i}\right)
\end{aligned}
$$




$$
\begin{aligned}
& +\frac{(\Delta t)^{2}}{3}\left(P_{2}^{0} z_{i, 0}+P_{2}^{N} z_{i, N}+\sum_{l=1,3}^{N-1} P_{2}^{l} z_{i, l}+\sum_{l=2,4}^{N-2} P_{2}^{l} z_{i, l}\right) \\
& +\frac{(\Delta t)^{2}}{2}\left(s_{i, 1}+s_{i, 0}\right), \quad i=1(1) M-1 .
\end{aligned}
$$

\section{Stability analysis}

The von Neumann stability $[8,9,22,23,28,30]$ is carried out for the discretized system of hyperbolic wave equation.

Theorem 5 Show that the proposed numerical scheme is stable.

Proof Taking $s(r, t)=0$ and assuming local constant $\alpha_{j}=\alpha_{j+1}=\bar{b}_{1}$ for known level in (12), we get

$$
\begin{aligned}
& -A z_{i-1, j+1}+(1+2 A-B) z_{i, j+1}-A z_{i+1, j+1} \\
& =A z_{i-1, j}+(2+2 A+B) z_{i, j}+A z_{i+1, j}-z_{i, j-1},
\end{aligned}
$$

where

$$
B=\frac{(\Delta t)^{2}}{2} \bar{b}_{1}
$$

Now we consider one Fourier mode out of the full solution $z_{i, j}=\hat{z}^{j} e^{i \omega \phi}$ as trial solutions at a given point $r_{i}$, where $\phi=\theta h$ is the phase angle, $i$ is the node number, and $\omega=\sqrt{-1}$. Using trial solutions in the above equation and simplifying the terms, we get

$$
(-2 A \cos \phi+(1+2 A-B)) \hat{z}^{2}-(2 A \cos \phi+(2+2 A+B)) \hat{z}+1=0
$$

which can be written as

$$
\beta_{1} \hat{z}^{2}-\beta_{2} \hat{z}+\beta_{3}=0
$$

where

$$
\beta_{1}=-2 A \cos \phi+(1+2 A-B), \quad \beta_{2}=2 A \cos \phi+(2+2 A+B), \quad \beta_{3}=1 .
$$

Under the transformation $\hat{z}=\frac{1+\rho}{1-\rho}$ in equation (24), we get

$$
\left(\beta_{1}+\beta_{2}+\beta_{3}\right) \rho^{2}+2\left(\beta_{1}-\beta_{3}\right) \rho+\left(\beta_{1}-\beta_{2}+\beta_{3}\right)=0 .
$$

The discretized system (22) will be stable if

$$
\beta_{1}+\beta_{2}+\beta_{3} \geq 0, \quad \beta_{1}-\beta_{3} \geq 0, \quad \beta_{1}-\beta_{2}+\beta_{3} \geq 0 .
$$

Using the values of $\beta_{1}, \beta_{2}, \beta_{3}$ and simplifying the terms, we get

$$
\beta_{1}+\beta_{2}+\beta_{3}=\frac{2(\Delta t)^{2}}{(\Delta r)^{2}}+3
$$




$$
\begin{aligned}
& \beta_{1}-\beta_{3}=\frac{(\Delta t)^{2}}{(\Delta r)^{2}}\left(2 \sin ^{2}\left(\frac{\phi}{2}\right)-(\Delta r)^{2} \bar{b}_{1}\right) \\
& \beta_{1}-\beta_{2}+\beta_{3}=2+\frac{2(\Delta t)^{2}}{(\Delta r)^{2}}\left(2 \sin ^{2}\left(\frac{\phi}{2}\right)-\frac{(\Delta r)^{2}}{2} \bar{b}_{1}-1\right) .
\end{aligned}
$$

It is clear from (27) that $\beta_{1}+\beta_{2}+\beta_{3} \geq 0$. From (28) and (29), we get $\beta_{1}-\beta_{3} \geq 0$ and $\beta_{1}-$ $\beta_{2}+\beta_{3} \geq 0$ if $(\Delta r)^{2} \leq \frac{1}{\bar{b}_{1}}\left(2 \sin ^{2}\left(\frac{\phi}{2}\right)-1\right)$, and the discretized system of (1) will be stable.

\section{Numerical solution of the inverse problem}

In this section, we want to find accurate and stable identification of $z(r, t)$ and $\alpha(t)$ which satisfies the nonlinear and ill-posed inverse problem (1)-(5). This is achieved by minimizing the objective function

$$
\mathbb{F}(\alpha)=\left\|z\left(R_{0}, t\right)-q(t)\right\|_{L^{2}[0, T]}^{2},
$$

where $z$ solves the forward problem (1)-(4) for given $\alpha(t)$. The discrete form of equation (30) is

$$
\mathbb{F}(\underline{\alpha})=\sum_{j=1}^{N}\left[w\left(R_{0}, t_{j}\right)-q\left(t_{j}\right)\right]^{2} .
$$

The objective function $\mathbb{F}$ (31) is minimized by the MATLAB subroutine lsqnonlin [21].

\section{Numerical results and discussion}

The accuracy is measured by RMS:

$$
\begin{aligned}
& \operatorname{RMS}(\alpha)=\left[\frac{T}{N} \sum_{j=1}^{N}\left(\alpha^{\text {numerical }}\left(t_{j}\right)-\alpha^{\text {exact }}\left(t_{j}\right)\right)^{2}\right]^{1 / 2}, \\
& \operatorname{RMS}(q)=\left[\frac{T}{N} \sum_{j=1}^{N}\left(q^{\text {numerical }}\left(t_{j}\right)-q^{\text {exact }}\left(t_{j}\right)\right)^{2}\right]^{1 / 2} .
\end{aligned}
$$

Here, for simplicity, we take $T=1$, and $10^{2}$ and $-10^{2}$ as upper and lower bounds, respectively, for coefficient $\alpha(t)$.

The inverse problem (1)-(5) is solved with exact and noisy measurement data (5). We numerically simulated the perturbed data as follows:

$$
q^{\epsilon}\left(t_{j}\right)=q\left(t_{j}\right)+\epsilon_{j}, \quad j=0(1) N
$$

where $\epsilon_{j}$ are random variables with mean zero and standard deviation $\sigma$ given by

$$
\sigma=p \times \max _{t \in[0, T]}|q(t)|,
$$

where $p$ denotes the percentage of noise. In the case of noisy data (34), we replace $q\left(t_{j}\right)$ with $q^{\epsilon}\left(t_{j}\right)$ in (31). 


\subsection{Test 1}

The proposed problem (1)-(5) is considered with some smooth potential term

$$
\alpha(t)=-1-\pi t, \quad t \in[0,1]
$$

the exact solution

$$
z(r, t)=\exp (-1-t) \cos (2 \pi r), \quad(r, t) \in \bar{D}_{T},
$$

and the rest of the data are as follows:

$$
\begin{aligned}
& \int_{0}^{1} z(r, t) d r=0, \quad P_{1}(t)=0, \quad P_{2}(t)=0, \quad R_{0}=0.5, \\
& \xi(r)=\exp (-1) \cos (2 \pi r), \\
& \zeta(r)=-\exp (-1) \cos (2 \pi r), \quad s(r, t)=\exp (-1-t)\left(2+4 \pi^{2}+\pi t\right) \cos (2 \pi r) .
\end{aligned}
$$

The periodic $\mathrm{BCs}$

$$
z(0, t)=\exp (-1-t)=z(1, t), \quad t \in[0,1]
$$

and the over-determination condition

$$
q(t)=z\left(R_{0}, t\right)=-\exp (-1-t), \quad t \in[0,1]
$$

It is observed that Theorem 3 guarantees the uniqueness of the solution because their conditions have been fulfilled. The accuracy of problem (1)-(4) has been assessed with data (37) and (38) when $\alpha(t)$ is given by (36). Fig. 1 depicts the approximate displacement measurement in (5) in comparison to the analytical solution (40) obtained by using the Crank-Nicolson FDM with $M=N \in\{10,20,40,80\}$. The exact (37) and approximate solutions for the displacement $z(r, t)$ are illustrated in Fig. 2. The absolute errors between

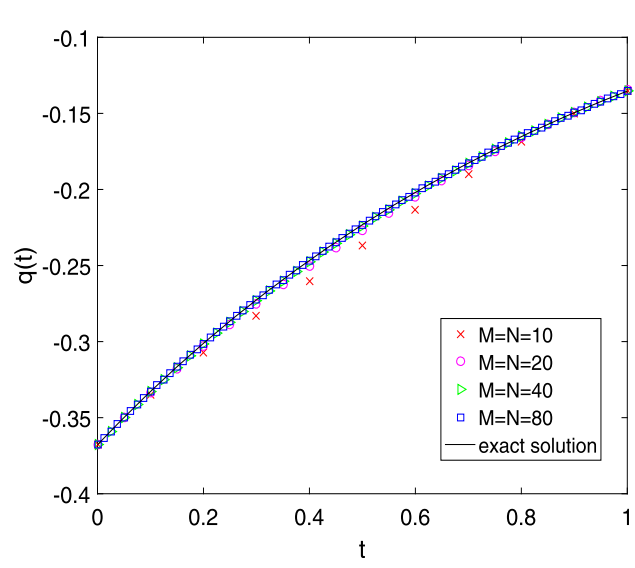

Figure 1 The approximate and exact (40) $q(t)$ for the forward problem 

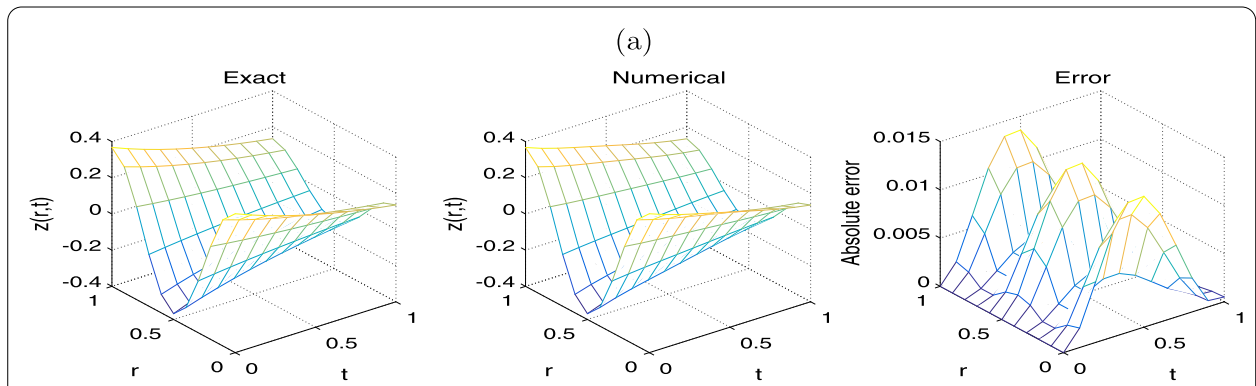

(b)
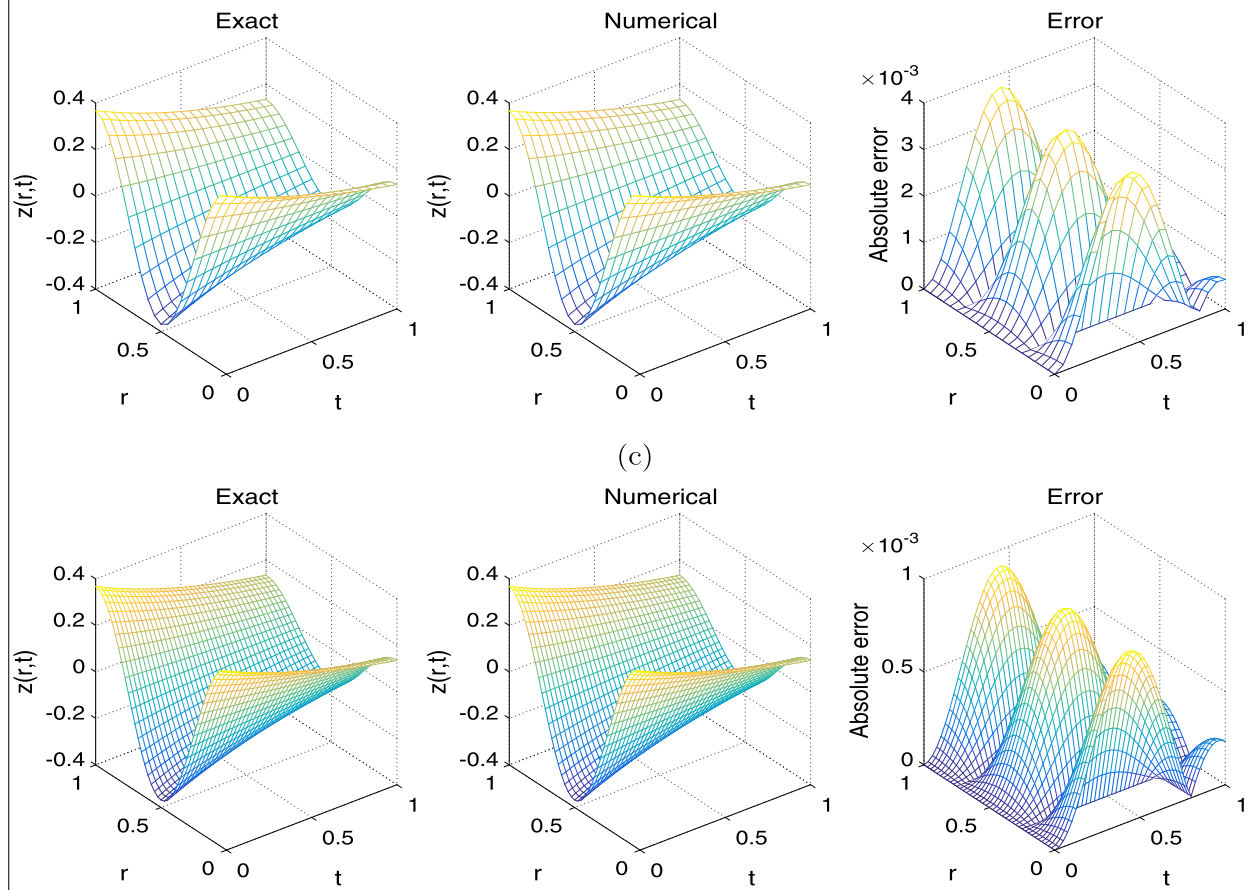

(c)
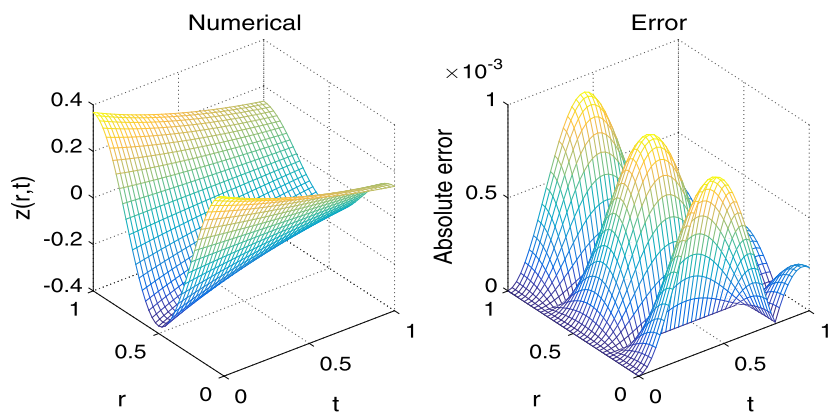

(d)
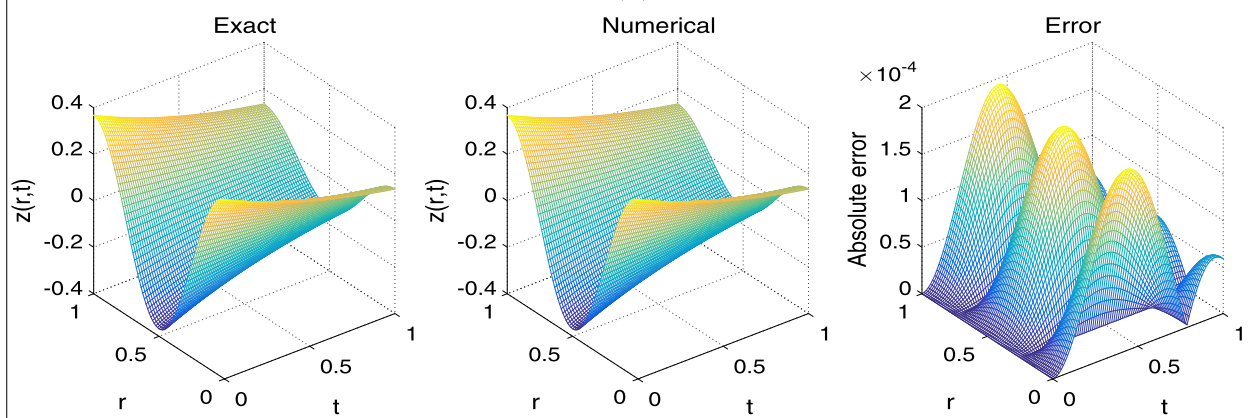

Figure 2 The exact (37) and approximate $z(r, t)$, and absolute errors with various grid sizes $M=N$ : (a) 10 , (b) 20, (c) 40, and (d) 80 for the forward problem

those solutions are also included and it can be seen that these errors decrease as the $\mathrm{CN}$ FDM grid becomes finer. A good agreement observed between the analytical (40) and the approximate $q(t)$ solutions as the mesh size decreases, see Table 1 .

Next, we fix $\Delta r=\Delta t=0.025$, and the time-dependent potential coefficient $\alpha(t)$ determination is started, when the value of $p$ equal zero in equation (5), as put in (35). The 
Table 1 The RMS error norm for direct problem

\begin{tabular}{lllll}
\hline$M=N$ & 10 & 20 & 40 & 80 \\
\hline RMS $(q)$ & $8.5 \mathrm{E}-3$ & $2.2 \mathrm{E}-3$ & $5.3 \mathrm{E}-4$ & $1.1 \mathrm{E}-4$ \\
\hline
\end{tabular}

(a)

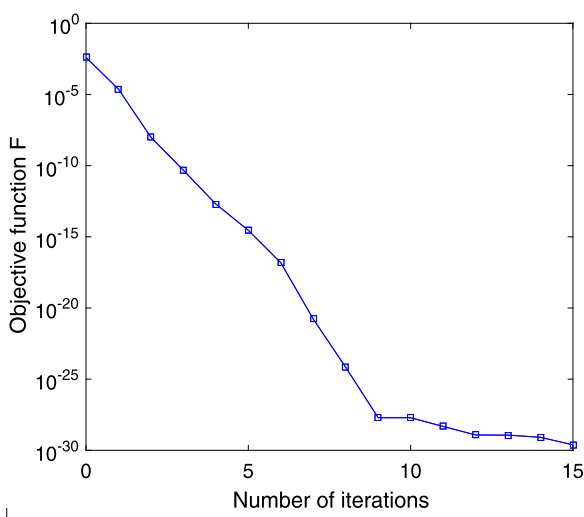

(b)

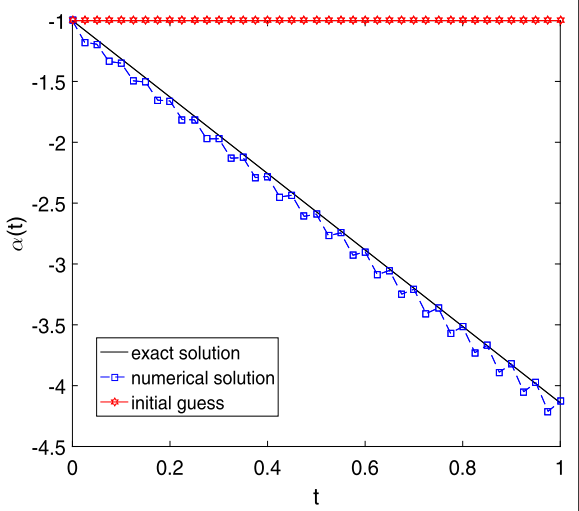

Figure 3 (a) $\mathbb{F}(31)$, (b) the exact (36) and approximate $\alpha(t)$ with $p=0$ (Test 1)

initial guess (IG) for $\underline{\alpha}$ is taken as follows:

$$
\alpha^{0}\left(t_{j}\right)=\alpha(0)=-1, \quad j=1(1) N
$$

Fig. 3(a) is depicted by the objective function $\mathbb{F}(31)$, and a monotonically decreasing convergence is observed by making 15 time repetitions for attaining a less order tolerance $O\left(10^{-30}\right)$. Numerical outcomes for the potential $\alpha(t)$ are observed in Fig. 3(b) and also obtaining with $\operatorname{RMS}(\alpha)=0.0868$. The perturbed data has been used to examine the stability of the approximate solution. The noise $p \in\left\{\frac{1}{100} \%, \frac{1}{10} \%\right\}$ is included to simulate the input noisy data via equation (34) for $q(t)$. The potential term $\alpha(t)$ is depicted in Figs. 4 and 5 . From Figs. 4(a) and 5(a) it can be observed that as $p$ is increased the approximate results for $\alpha(t)$ start to build up oscillations. To retrieve stability, we penalize $\mathbb{F}$ (30) by adding $\beta\|\alpha(t)\|_{L^{2}[0, T]}^{2}$ to it, where $\beta>0$ is Tikhonov's regularization parameter. Then a discretized form of Tikhonov functional is

$$
\mathbb{F}_{\beta}(\underline{\alpha})=\mathbb{F}(\underline{\alpha})+\beta \sum_{j=1}^{N} \alpha_{j}^{2} .
$$

For $p \in\{0.01 \%, 0.1 \%\}$ noise, Figs. 4(b) and 5(b) show the analytical (36) and the approximate $\alpha(t)$ achieved by minimizing $\mathbb{F}_{\beta}$ (42) for various $\beta$. The $\operatorname{RMS}(\alpha)$ values are $\{3.1204,0.0790,0.0776\}$ for the value of $p=\frac{1}{100} \%$, and $\{34.2297,0.1573,0.1100\}$ for the value of $p=\frac{1}{10} \%$, respectively, with $\beta \in\left\{0,10^{-10}, 10^{-9}\right\}$ and $\beta \in\left\{0,10^{-9}, 10^{-8}\right\}$. It is noticed that the approximate $\alpha(t)$ obtained with $\beta=0$ demonstrates instability; however, on inclusion of regularization with $\beta=10^{-10}$ to $10^{-8}$, a stable solution is obtained which is consistent in accuracy with $p=0.01 \%$ and $0.1 \%$ violating the input data (34). The absolute error norms between the exact (37) and approximate solutions with $\beta=0,10^{-9}$ 
(a)

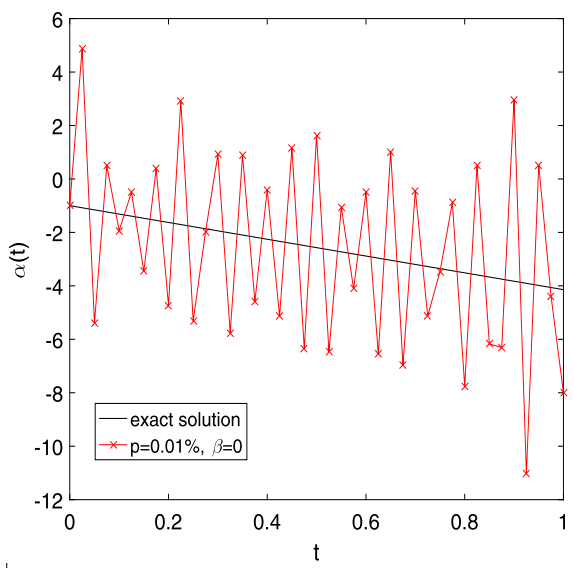

(b)

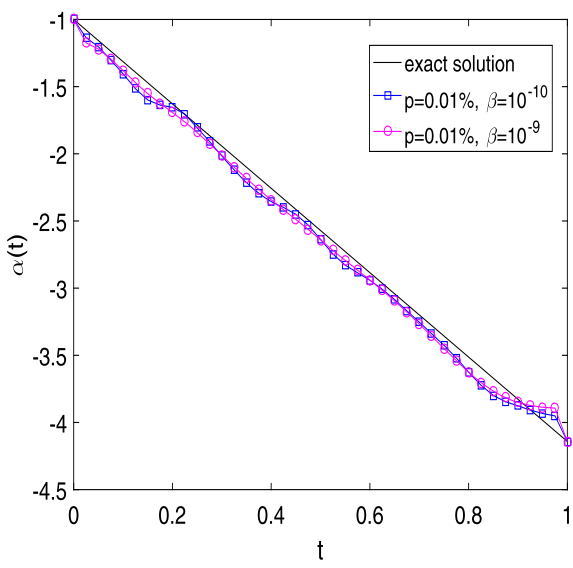

Figure 4 The analytical (36) and approximate $\alpha(t)$ with $p=0.01 \%$ for: (a) $\beta=0$ and (b) $\beta=10^{-10}$ and $10^{-9}$ for Test 1

(a)

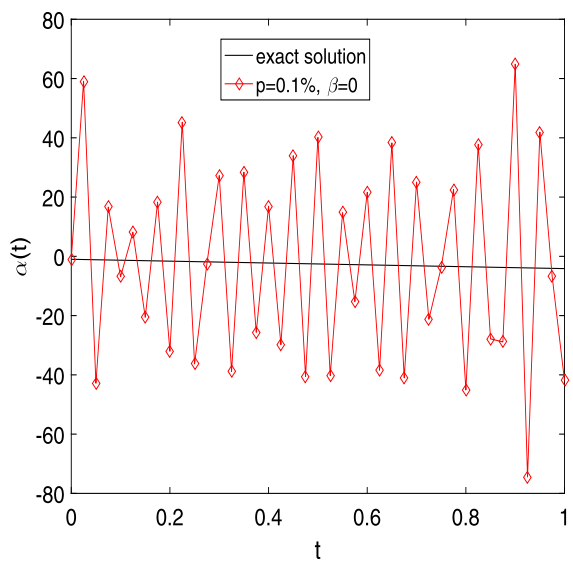

(b)

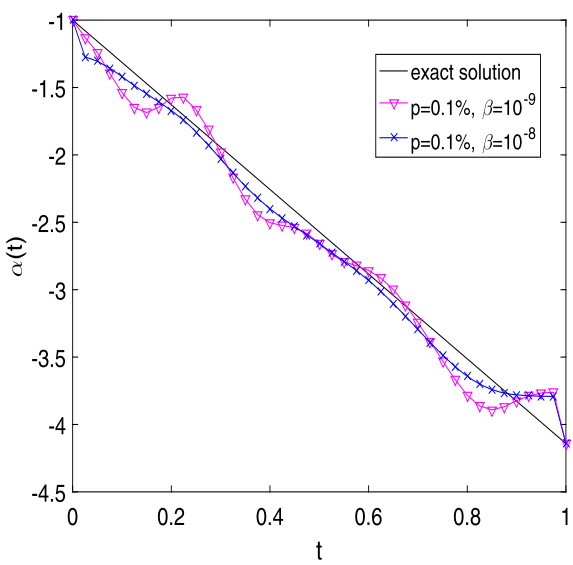

Figure 5 The analytical (36) and approximate $\alpha(t)$ with $p=0.1 \%$ for: (a) $\beta=0$ and (b) $\beta=10^{-9}$ and $10^{-8}$ for Test 1

and $10^{-8}$ are illustrated in Fig. 6 . It is observed that the displacement $z(r, t)$ component is accurate and stable when penalty term $\beta>0$ is added as in (42) to stabilize the solution.

\subsection{Test 2}

The smooth time-wise potential $\alpha(t)$ given by (36) has been recovered in Test 1 . Now, consider a nonlinear numerical test problem which is given by

$$
\begin{aligned}
& \alpha(t)=-\exp (t)-\pi \cos ^{2}(3 \pi t), \quad t \in[0,1], \\
& s(r, t)=\exp (-1-t)\left(1+\exp (t)+4 \pi^{2}+\pi \cos ^{2}(3 \pi t)\right) \cos (2 \pi r) .
\end{aligned}
$$


(a)

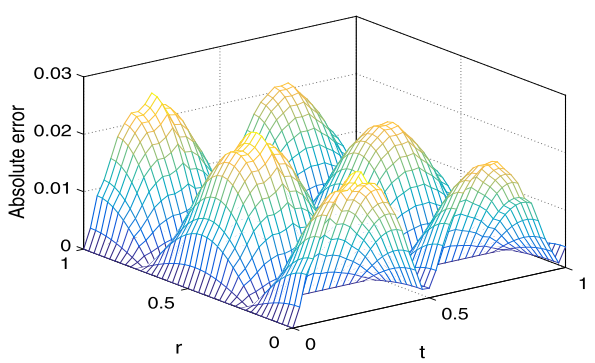

(b)

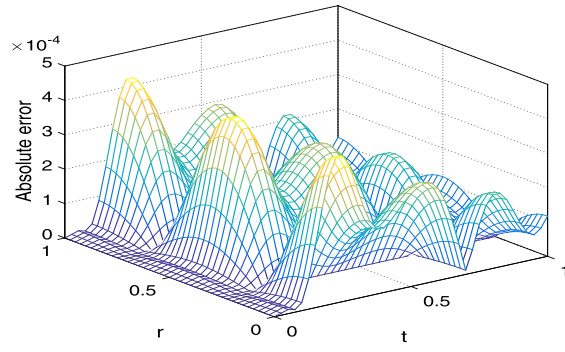

(c)

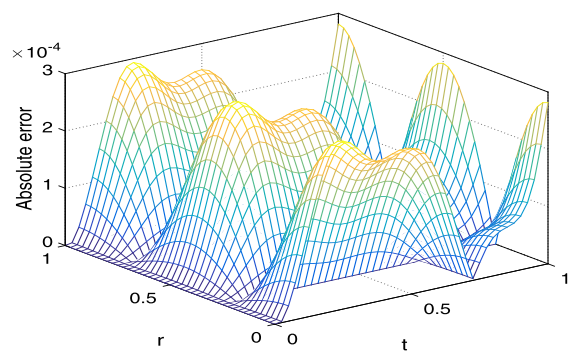

Figure 6 The absolute errors between the analytical (37) and approximate $z(r, t)$ with $\beta:(\mathbf{a}) 0,(\mathbf{b}) 10^{-9}$, and (c) $10^{-8}$ for $p=0.1 \%$ (Test 1)

(a)

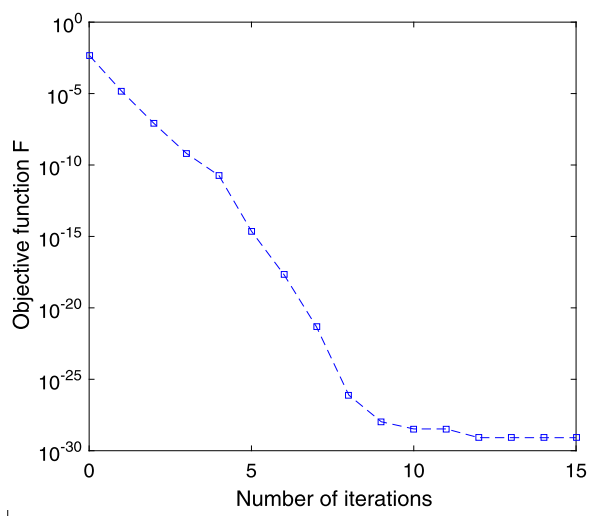

(b)

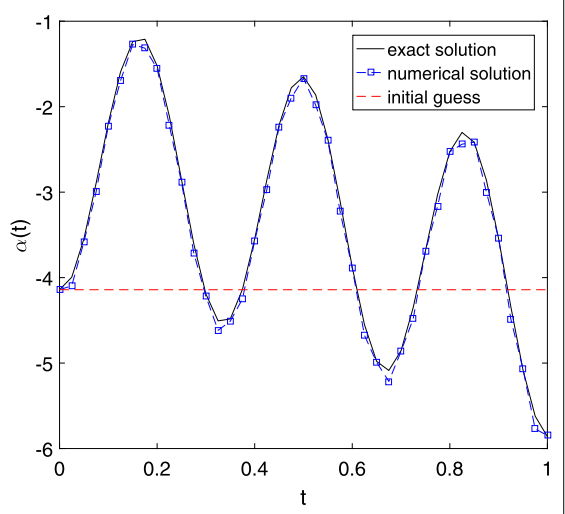

Figure 7 (a) $\mathbb{F}(31)$, (b) the analytical (43) and approximate $\alpha(t)$ with $p=0$ for Test 2

Since the conditions of Theorem 3 hold, the solution uniqueness is guaranteed. The IG for $\underline{\alpha}(t)$ is considered as follows:

$$
\alpha^{0}\left(t_{j}\right)=\alpha(0)=-1-\pi, \quad j=1(1) N .
$$

We take fixed values $M=N=40$ as in the previous test problem, and value of $p=0$ in $q(t)$, as also in equation (35). Fig. 7(a) is depicted by the objective function $\mathbb{F}(31)$, and a monotonically decreasing convergence is observed by making 15 time repetitions for 
(a)

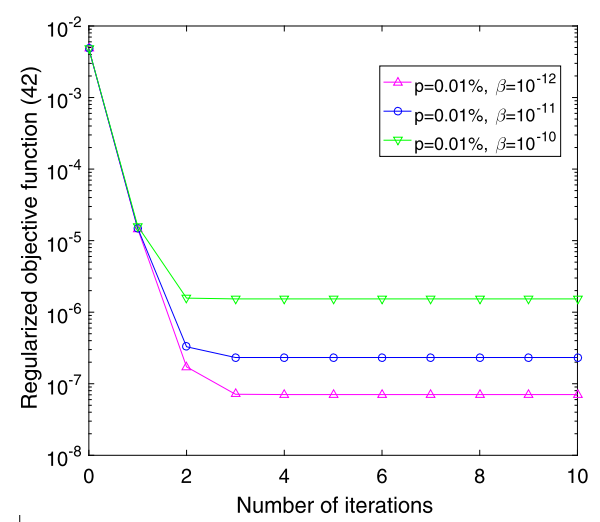

(b)

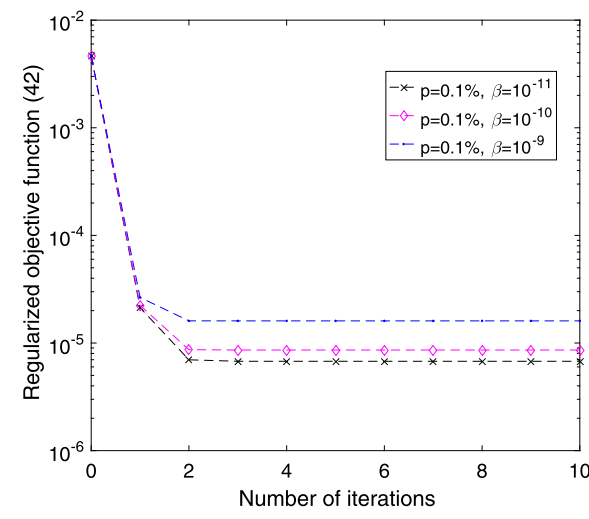

Figure 8 The regularized function $\mathbb{F}_{\beta}$ (42) with $p$ (a) $0.01 \%$, (b) $0.1 \%$ for Test 2

(a)

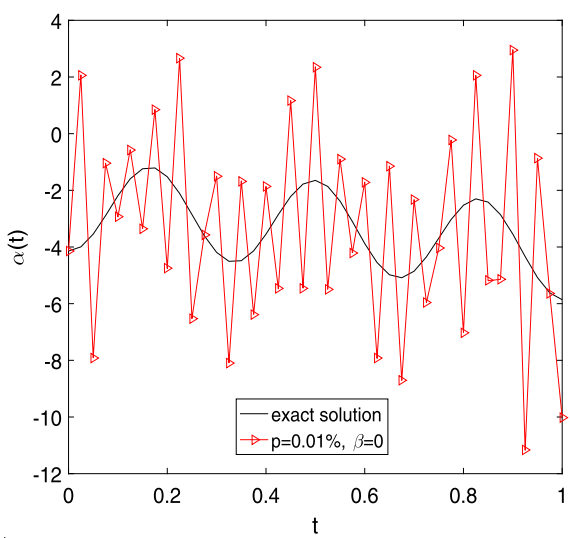

(b)

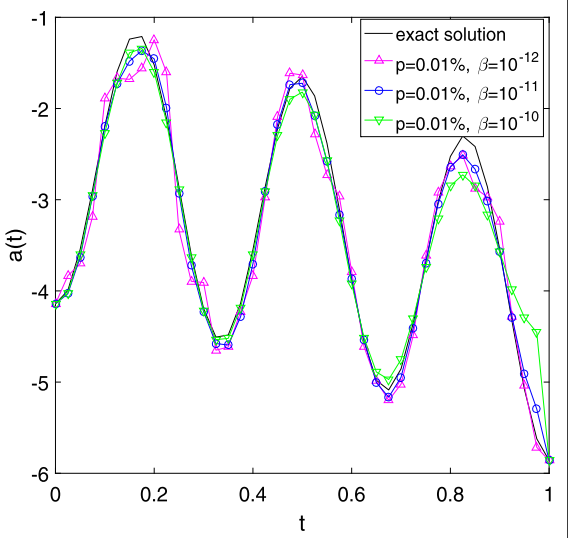

Figure 9 The analytical (43) and approximate $\alpha(t)$ with $p=0.01 \%$ for $\beta$ : (a) 0 and (b) $10^{-12}, 10^{-11}, 10^{-10}$ for Test 2

attaining a less order tolerance $O\left(10^{-30}\right)$. Numerical outcomes for the potential $\alpha(t)$ are observed in Fig. 7(b) and also obtaining with $\operatorname{RMS}(\alpha)=0.0872$.

The $\mathbb{F}$ (31) is depicted in Fig. 7(a), where a monotonically decreasing convergence is achieved in 15 iterations for achieving $O\left(10^{-30}\right)$. Fig. 7(b) depicts the exact (43) and approximate $\alpha(t)$, obtaining with $\operatorname{RMS}(\alpha)=0.0872$.

Next, we take $p \in\left\{\frac{1}{100} \%, \frac{1}{10} \%\right\}$ to the measured data $q(t)$, as also in equation (35), for checking the stability of problem. The regularized function $\mathbb{F}_{\beta}(42)$ is depicted in Fig. 8 with various parameters. The approximate outcomes of potential $\alpha(t)$ are illustrated in Figs. 9 and 10. As in the previous test problem, it is observed that the numerical solutions for $\beta=0$ tabulated in Figs. 9(a) and 10(a) are unstable due to high oscillations and unbounded, obtaining with $\operatorname{RMS}(\alpha)=3.3676$ for the value of $p=\frac{1}{100} \%$ and $\operatorname{RMS}(\alpha)=$ 33.9465 for the value of $p=\frac{1}{10} \%$. However, the addition of some restrictions on $\beta>0$ in $\mathbb{F}_{\beta}(42)$ improves the stability of the numerical solutions, as drawn further in Figs. 9 (b) and 10 (b), respectively. It is observed that the addition of $\beta \in\left\{10^{-12}, 10^{-11}, 10^{-10}\right\}$ for $\frac{1}{100} \%$ and 
(a)

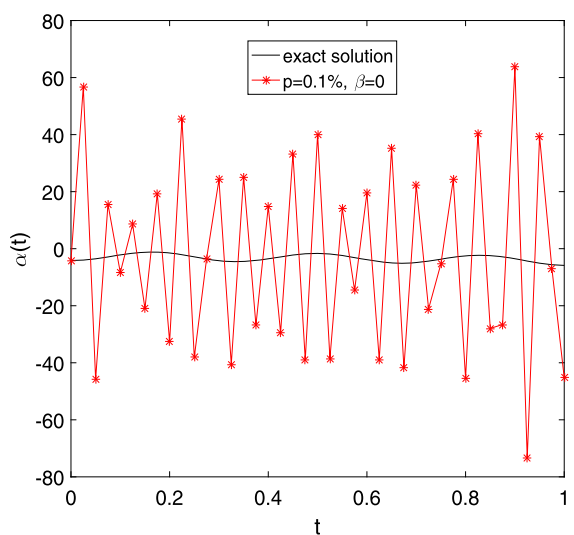

(b)

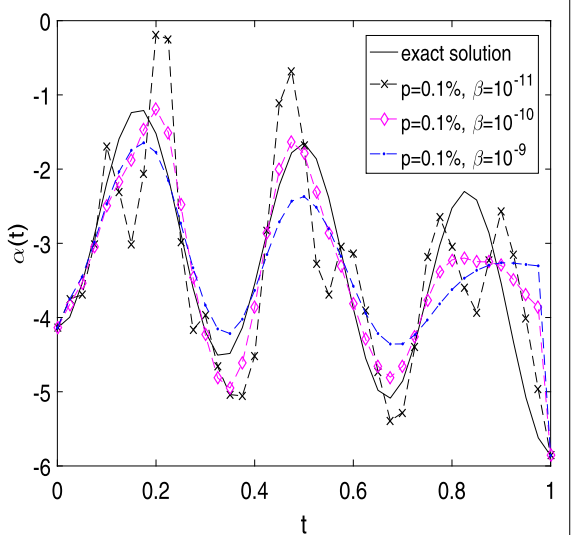

Figure 10 The analytical (43) and approximate $\alpha(t)$ with $p=0.1 \%$ for $\beta$ : (a) 0 and (b) $10^{-11}, 10^{-10}, 10^{-9}$ for Test 2

(a)

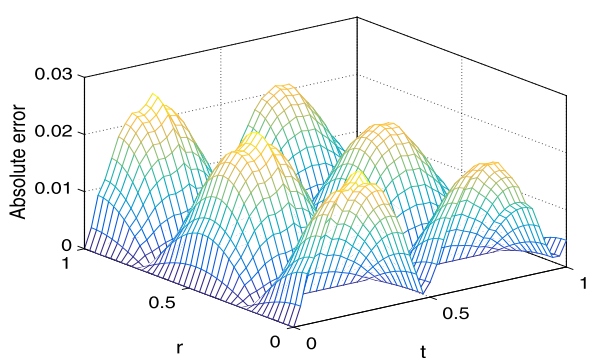

(c)

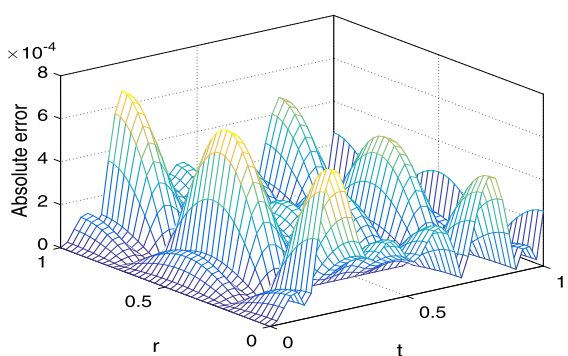

(b)

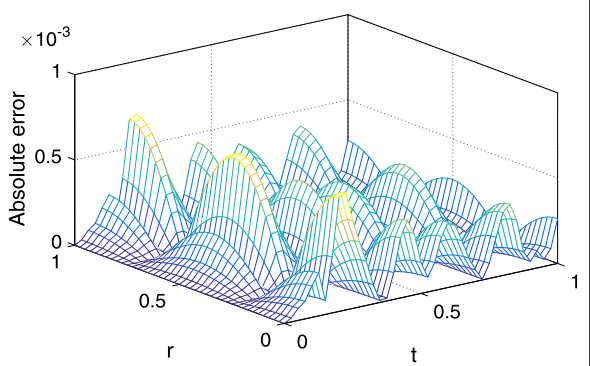

(d)

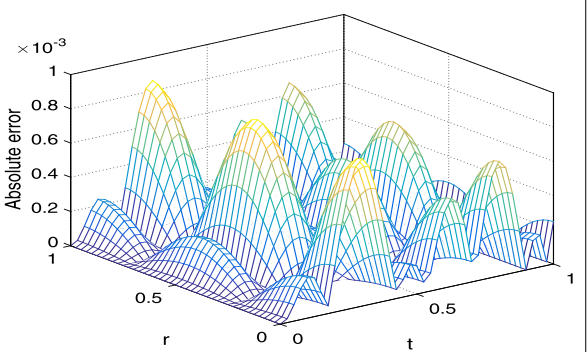

Figure 11 The absolute errors between the exact (37) and approximate $z(r, t)$ with $\beta:$ : (a) 0 , (b) $10^{-11}$, (c) $10^{-10}$, and (d) $10^{-9}$ for $p=0.1 \%$, for Test 2

$\beta \in\left\{10^{-11}, 10^{-10}, 10^{-9}\right\}$ for $\frac{1}{10} \%$ provides a perfect and stable approximate solution for $\alpha(t)$, getting with $\operatorname{RMS}(\alpha) \in\{0.2368,0.1245,0.2728\}$ and $\operatorname{RMS}(\alpha) \in\{0.8454,0.5282,0.6936\}$. The comparison between analytical (37) and approximate $z(r, t)$ in terms of absolute errors with and without descriptor parameters are depicted in Fig. 11, where $\beta>0$ has the effect of reducing the unstable performance of the restored displacement. Refer to Table 2 for additional information on the $\operatorname{RMS}(\alpha)$ values, the minimum value of $\mathbb{F}$ or $\mathbb{F}_{\beta}$ at final 
Table 2 The RMS values (32) for $p \in\{0,0.01 \%, 0.1 \%\}$ and $\beta \in\left\{0,10^{-12}, 10^{-11}, 10^{-10}, 10^{-9}, 10^{-8}, 10^{-7}\right\}$ for Tests 1 and 2

\begin{tabular}{|c|c|c|c|c|c|c|c|}
\hline \multicolumn{4}{|l|}{ Test 1} & \multicolumn{4}{|l|}{ Test 2} \\
\hline$p$ & $\beta$ & $\operatorname{RMS}(\alpha)$ & $\begin{array}{l}\text { Minimum value } \\
\text { of } \mathbb{F} \text { or } \mathbb{F}_{\beta}\end{array}$ & $p$ & $\beta$ & $\operatorname{RMS}(\alpha)$ & $\begin{array}{l}\text { Minimum value } \\
\text { of } \mathbb{F} \text { or } \mathbb{F}_{\beta}\end{array}$ \\
\hline 0 & 0 & 0.0868 & $\mathbb{F}=2.4 \mathrm{E}-30$ & 0 & 0 & 0.0872 & $\mathbb{F}=8.1 \mathrm{E}-30$ \\
\hline $0.01 \%$ & $\begin{array}{l}0 \\
10^{-10} \\
10^{-9} \\
10^{-8}\end{array}$ & $\begin{array}{l}3.4204 \\
0.0790 \\
0.0776 \\
0.0982\end{array}$ & $\begin{array}{l}\mathbb{F}=1.3 \mathrm{E}-29 \\
\mathbb{F}_{\beta}=1.1 \mathrm{E}-7 \\
\mathbb{F}_{\beta}=4.2 \mathrm{E}-7 \\
\mathbb{F}_{\beta}=3.2 \mathrm{E}-6\end{array}$ & $0.01 \%$ & $\begin{array}{l}0 \\
10^{-12} \\
10^{-11} \\
10^{-10}\end{array}$ & $\begin{array}{l}3.3676 \\
0.2368 \\
0.1245 \\
0.2728\end{array}$ & $\begin{array}{l}\mathbb{F}=8.5 \mathrm{E}-30 \\
\mathbb{F}_{\beta}=7.1 \mathrm{E}-8 \\
\mathbb{F}_{\beta}=2.3 \mathrm{E}-7 \\
\mathbb{F}_{\beta}=1.5 \mathrm{E}-6\end{array}$ \\
\hline $0.1 \%$ & $\begin{array}{l}0 \\
10^{-9} \\
10^{-8} \\
10^{-7}\end{array}$ & $\begin{array}{r}34.2297 \\
0.1573 \\
0.1100 \\
0.1701\end{array}$ & $\begin{array}{l}\mathbb{F}=1.3 \mathrm{E}-29 \\
\mathbb{F}_{\beta}=8.4 \mathrm{E}-6 \\
\mathbb{F}_{\beta}=1.1 \mathrm{E}-5 \\
\mathbb{F}_{\beta}=3.6 \mathrm{E}-5\end{array}$ & $0.1 \%$ & $\begin{array}{l}0 \\
10^{-11} \\
10^{-10} \\
10^{-9}\end{array}$ & $\begin{array}{r}33.9465 \\
0.8454 \\
0.5282 \\
0.6936\end{array}$ & $\begin{array}{l}\mathbb{F}=2.1 \mathrm{E}-29 \\
\mathbb{F}_{\beta}=6.7 \mathrm{E}-6 \\
\mathbb{F}_{\beta}=8.6 \mathrm{E}-6 \\
\mathbb{F}_{\beta}=1.6 \mathrm{E}-5\end{array}$ \\
\hline
\end{tabular}

iteration without and with regularization for both numerical test problems. For the term $\alpha(t)$, the same results may be derived regarding the stable reconstruction.

\section{Concluding remarks}

For the first time, the reconstruction of a potential coefficient $\alpha(t)$ and the displacement distribution $z(r, t)$ from the over-determination condition in the hyperbolic equation using nonlocal ICs and periodic BCs have been computationally addressed. The CN-FDM was applied to solve the direct problem. The stability analysis of the discretized system of the wave equation has been discussed using the von Neumann method. The TR was used to overcome the instability due to the ill-posed issue. For both numerical test problems, the RMS values for noise without and with regularization were compared. It has been noticed that on the inclusion of regularization with $\beta=10^{-12}$ to $\beta=10^{-7}$, a stable solution is obtained which is consistent in accuracy with $0.01 \%$ and $0.1 \%$ noise. Finally, future study into the extension of the suggested approach for determining the time-wise potential coefficient in a two-dimensional wave equation is an intriguing prospect.

Acknowledgements

The authors are indebted to the anonymous referees for their valuable comments and suggestions that helped improve the paper. Also, we thank Dr Muhammad Amin for his assistance in proofreading of the manuscript.

Funding

No external funding is available for this research.

Availability of data and materials

Not applicable.

\section{Declarations}

Competing interests

The authors declare that they have no competing interests.

Authors' contributions

All authors equally contributed to this work. All authors read and approved the final manuscript.

\section{Author details}

${ }^{1}$ Department of Mathematics, Faculty of Science, Jazan University, Jazan, Saudi Arabia. ${ }^{2}$ Department of Mathematics, University of Sargodha, 40100 Sargodha, Pakistan. ${ }^{3}$ Department of Mathematics, Faculty of Arts and Sciences, Çankaya University, 06530 Ankara, Turkey. ${ }^{4}$ Institute of Space Sciences, P.O. Box, MG-23, R 76900, Magurele-Bucharest, Romania.

${ }^{5}$ Department of Medical Research, China Medical University Hospital, China Medical University, Taichung, Taiwan. 


\section{Publisher's Note}

Springer Nature remains neutral with regard to jurisdictional claims in published maps and institutional affiliations.

\section{Received: 29 August 2021 Accepted: 29 September 2021 Published online: 15 October 2021}

\section{References}

1. Azizbayov, E.I.: On the unique recovery of time-dependent coefficient in a hyperbolic equation from nonlocal data. UPB Sci. Bull., Ser. A 82, 171-182 (2020)

2. Bakushinsky, A.B., Leonov, A.S.: Fast numerical method of solving 3D coefficient inverse problem for wave equation with integral data. J. Inverse III-Posed Probl. 26, 477-492 (2018)

3. Bellassoued, M., Yamamoto, M.: Determination of a coefficient in the wave equation with a single measurement. Appl. Anal. 87, 901-920 (2008)

4. Boumenir, A., Tuan, V.K.: An inverse problem for the wave equation. J. Inverse IIl-Posed Probl. 19, 573-592 (2011)

5. Bui, A.T.: An inverse source problem for the wave equation. Nonlinear Anal., Theory Methods Appl. 55, 269-284 (2003)

6. Cannon, J.R., DuChateau, P.: An inverse problem for an unknown source term in a wave equation. SIAM J. Appl. Math. 43, 553-564 (1983)

7. Cannon, J.R., Dunninger, D.R.: Determination of an unknown forcing function in a hyperbolic equation from overspecified data. Ann. Mat. Pura Appl. 1, 49-62 (1970)

8. Chattaraj, P., Rao Koneru, S., Deb, B.: Stability analysis of finite difference schemes for quantum mechanical equations of motion. J. Comput. Phys. 72, 504-512 (1987)

9. Dhiman, N., Huntul, M.J., Tamsir, M.: A modified trigonometric cubic B-spline collocation technique for solving the time-fractional diffusion equation. Eng. Comput. (2021). https://doi.org/10.1108/EC-06-2020-0327

10. Eskin, G.: Inverse problems for general second order hyperbolic equations with time-dependent coefficients. Bull. Math. Sci. 7, 247-307 (2017)

11. Hasanov, H.A., Romanov, V.G.: Inverse problems for hyperbolic equations. In: Introduction to Inverse Problems for Differential Equations, pp. 123-143. Springer, Cham (2017)

12. Huntul, M.J.: Identifying an unknown heat source term in the third-order pseudo-parabolic equation from nonlocal integral observation. Int. Commun. Heat Mass Transf. 128, 105550 (2021)

13. Huntul, M.J.: Determination of a time-dependent potential in the higher-order pseudo-hyperbolic problem. Inverse Probl. Sci. Eng. (2021). https://doi.org/10.1080/17415977.2021.1964496

14. Huntul, M.J., Dhiman, N., Tamsir, M.: Reconstructing an unknown potential term in the third-order pseudo-parabolic problem. Comput. Appl. Math. 40, 140 (2021)

15. Huntul, M.J., Tamisr, M., Ahmadini, A.: An inverse problem of determining the time-dependent potential in a higher-order Boussinesq-Love equation from boundary data. Eng. Comput. (2021). https://doi.org/10.1108/EC-08-2020-0459

16. Huntul, M.J., Tamsir, M.: Recovery of timewise dependent heat source for a hyperbolic PDE from an integral condition. Math. Methods Appl. Sci. (2020). https://doi.org/10.1002/mma.6845

17. Huntul, M.J., Tamsir, M., Dhiman, N.: An inverse problem of identifying the time-dependent potential in a fourth-order pseudo-parabolic equation from additional condition. Numer. Methods Partial Differ. Equ. (2021). https://doi.org/10.1002/num.22778

18. Huntul, M.J., Tamsir, M., Dhiman, N.: Determination of a timewise potential in a wave equation with dynamic boundary condition from an additional measurement. Ain Shams Eng. J. 12, 3183-3193 (2021)

19. Hussein, S.O., Lesnic, D.: Determination of forcing functions in the wave equation. Part II: the time-dependent case. J. Eng. Math. 96, 135-153 (2015)

20. Jiang, D., Liu, Y., Yamamoto, M.: Inverse source problem for the hyperbolic equation with a time-dependent principal part. J. Differ. Equ. 262, 653-681 (2017)

21. Mathworks: Documentation optimization toolbox —least squares algorithms (2019). www.mathworks.com

22. Mittal, R.C., Jain, R.K.: B-splines methods with redefined basis functions for solving fourth order parabolic partial differential equations. Appl. Math. Comput. 217, 9741-9755 (2011)

23. O'Brien, G.G., Hyman, M.A., Kaplan, S.: A study of the numerical solution of partial differential equations. J. Math. Phys. 29, 223-251 (1950)

24. Ramm, A., Rakesh: Property C and an inverse problem for a hyperbolic equation. J. Math. Anal. Appl. 156, 209-219 (1991)

25. Salazar, R.: Determination of time-dependent coefficients for a hyperbolic inverse problem. Inverse Probl. 29, 095015 (2013)

26. Smith, G.D.: Numerical Solution of Partial Differential Equations: Finite Difference Methods, 3rd edn. Clarendon, Oxford (1985)

27. Stefanov, P., Uhlmann, G.: Recovery of a source term or a speed with one measurement and applications. Trans. Am. Math. Soc. 365, 5737-5758 (2013)

28. Tamsir, M., Dhiman, N., Chauhan, A., Chauhan, A.: Solution of parabolic PDEs by modified quintic B-spline Crank-Nicolson collocation method. Ain Shams Eng. J. (2020). https://doi.org/10.1016/j.asej.2020.08.028

29. Tekin, l.: Determination of a time-dependent coefficient in a wave equation with unusual boundary condition. Filomat 33, 2653-2665 (2019)

30. Vichnevetsky, R.: Stability charts in the numerical approximation of partial differential equations: a review. Math. Comput. Simul. 21, 170-177 (1979)

31. Yamamoto, M.: Stability, reconstruction formula and regularization for an inverse source hyperbolic problem by a control method. Inverse Probl. 11, 481-496 (1995)

32. Yang, L., Dehghan, M., Yu, J.N., Luo, G.W.: Inverse problem of time-dependent heat sources numerical reconstruction. Math. Comput. Simul. 81, 1656-1672 (2011) 